Document downloaded from:

http://hdl.handle.net/10251/102328

This paper must be cited as:

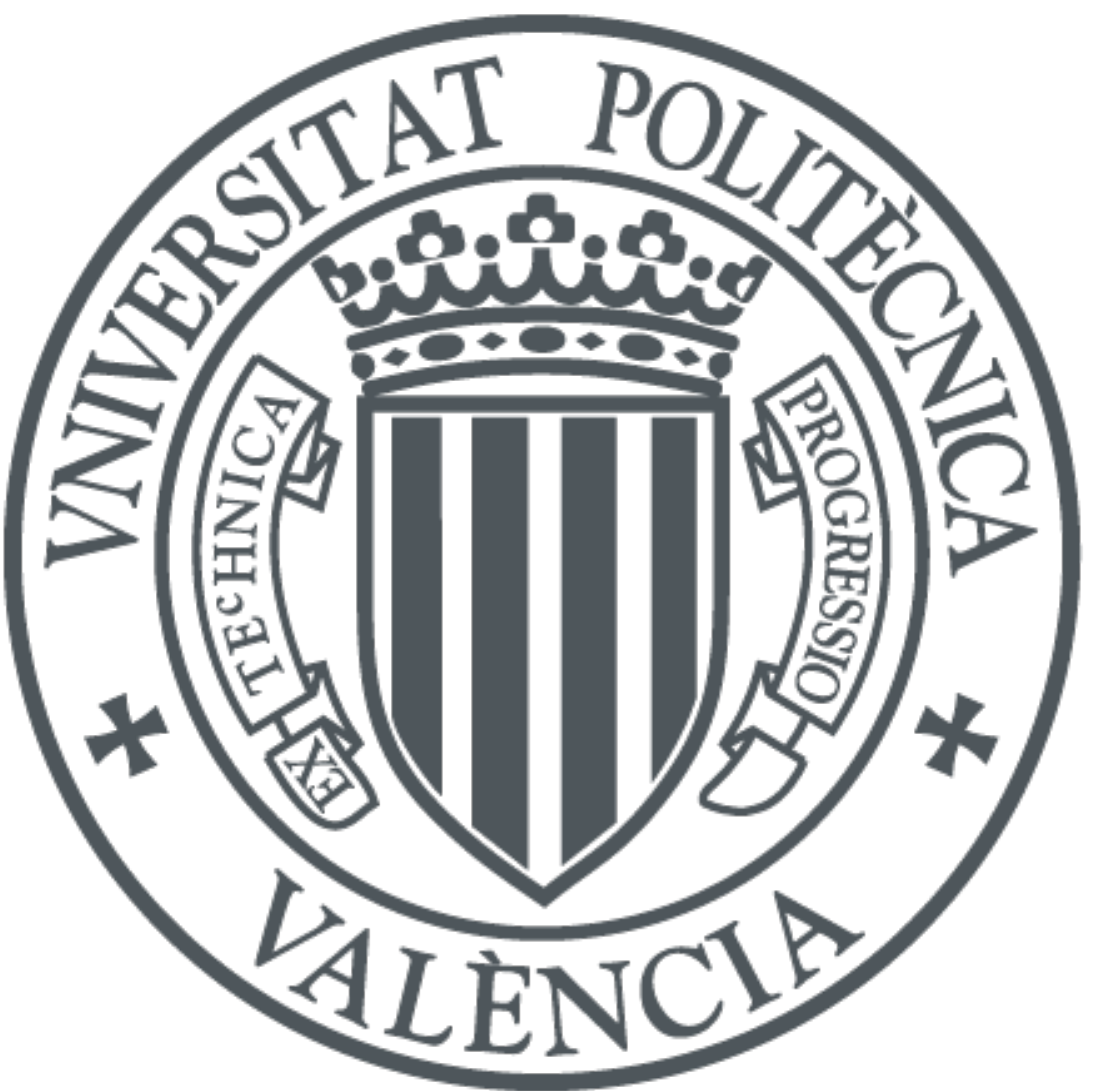

The final publication is available at

http://doi.org/10.1007/s13398-015-0272-0

Copyright Springer-Verlag

Additional Information 


\title{
Cauchyness and convergence in fuzzy metric spaces
}

\author{
Valentín Gregori, ${ }^{1}$ Juan-José Miñana, Samuel Morillas, \\ Almanzor Sapena ${ }^{2}$ \\ Instituto Universitario de Matemática Pura y Aplicada, Universitat Politècnica de \\ València, Camino de Vera s/n, 46022 Valencia (SPAIN).
}

\begin{abstract}
In this paper we survey some concepts of convergence and Cauchyness appeared separately in the context of fuzzy metric spaces in the sense of George and Veeramani. For each convergence (Cauchyness) concept we find a compatible Cauchyness (convergence) concept. We also study the relationship among them and the relationship with compactness and completeness (defined in a natural sense for each one of the Cauchy concepts). In particular, we prove that compactness implies $p$ completeness.
\end{abstract}

Key words: Fuzzy metric, Cauchy sequence, completeness, compactness. $M S C: 54 \mathrm{~A} 40,54 \mathrm{D} 35,54 \mathrm{E} 50$

\section{Introduction}

Kramosil and Michalek [19] gave a notion of fuzzy metric space, that we denote $K M$-fuzzy metric space, which could be considered as a reformulation, in the fuzzy context, of the notion of $P M$-space (or more precisely, Menger space). In [3] George and Veeramani introduced a concept of fuzzy metric space $(X, M, *)$ which is a slight modification of the $K M$-fuzzy metric space. Many concepts

\footnotetext{
* Corresponding author email: vgregori@mat.upv.es

1 Valentín Gregori acknowledges the support of Ministry of Economy and Competitiveness of Spain under grant MTM 2012-37894-C02-01

2 Almanzor Sapena acknowledges the support of Ministry of Economy and Competitiveness of Spain under grant TEC2013-45492-R.
} 
and results stating in both fuzzy settings come from the theory of $P M$-spaces. Now, for easy the reading and for simplicity, by fuzzy metric we mean a fuzzy metric in the sense of George and Veeramani and then all our results are established for them.

A fuzzy metric $M$ on a nonempty set $X$ induces a topology $\tau_{M}$ on $X$. As it was proved in [7] the class of topological spaces which are fuzzy metrizable (in its obvious sense) agrees with the class of metrizable spaces. A fuzzy metric space $(X, M, *)$, or simply $M$, is called complete if every Cauchy sequence (Definition 5) in $X$ is convergent.

The appearance of a parameter $t$ in the definition of $M$ allows to introduce new particular definitions in fuzzy metric context. In particular, the following well-motivated concepts (see the introductions of Sections 3-4) of convergence and Cauchyness related to sequences have appeared in the literature. In [6] M. Grabiec introduced a weaker concept than Cauchy sequence which we denote G-Cauchy (Definition 6). In [20] D. Mihet introduced a weaker concept than convergence denoted p-convergence (Definition 11). In [25] J.L. Ricarte and S. Romaguera introduced a stronger concept than Cauchy sequence (Definition 15). In [16] Gregori et al. introduced a stronger concept than convergence called $s$-convergence (Definition 18), and recently in [14] V. Gregori and J-J. Miñana have introduced a stronger concept than convergence called strong convergence. As it can be seen, all these concepts are related to the concept of Cauchy or convergent sequence.

If $(X, d)$ is a metric space then a sequence $\left\{x_{n}\right\}$ in $X$ is convergent to $x_{0}$ if and only if $\lim _{n} d\left(x_{n}, x_{0}\right)=0$ and $\left\{x_{n}\right\}$ is Cauchy if and only if $\lim _{n, m} d\left(x_{n}, x_{m}\right)=0$. Roughly speaking, one could say that the concept of Cauchyness is deduced from convergence replacing the simple limit (and removing $x_{0}$ ) by a double limit, or vice-versa. When extending this situation to the fuzzy setting we will say that the concept of Cauchyness is defined in a natural way from convergence, or vice-versa. Going back to the above concepts of convergence and Cauchyness, it seems natural to find and study its natural corresponding partner for each one of them. This idea was already suggested by D. Mihet [20]. So, the following two new concepts have appeared in the literature. In [10] V. Gregori et al. introduced the concept of $p$-Cauchy sequence (Definition 14) and in [24] S. Morillas and A. Sapena have introduced the concept of standard convergent sequence that we denote $s t d^{*}$-convergent sequence (Definition 16). Both concepts were deduced in a natural way from the corresponding ones as commented above. Surprisingly, the classical relation between convergence and Cauchyness was not accomplished in one case. Indeed, answering a question posted in [24], V. Gregori and J-J. Miñana proved in [15] the existence of $s t d^{*}$-convergent sequences which are not standard Cauchy. So it arises the first question: Does it always make sense to define a concept of A-convergence (respectively, A-Cauchyness) deduced in a natural way from 
a concept of $A$-Cauchyness (respectively, $A$-convergence)? In any case, which conditions should satisfy the pair $A$-convergence and $A$-Cauchyness in order to be considered appropriate? To approach these questions the authors in [15] introduced the concept of compatible pair (Definition 4). The case of the $G$-Cauchy concept is paradigmatic because one cannot obtain a concept of $G$ convergence deduced in a natural way from the $G$-Cauchy concept (see Section $3.2)$.

In this paper, for each one of the concepts above mentioned we discuss its appearance, interest, advantages and inconveniences. Further, we find for each Cauchyness concept (respectively, convergence concept) a compatible pair. Also we study the relationship among the concepts summarized in this paper. We also observe the relationship between compactness and each one of the concepts of completeness, which are defined in its obvious sense (Definition 35). In particular we prove that compactness implies $p$-completeness. Appropriate examples along the paper illustrate the theory.

The structure of the paper is as follows. Section 2 contains preliminaries. Section 3 contains the results for weaker concepts than convergence and Cauchyness. Section 4 contains the results for stronger concepts than convergence and Cauchyness. Section 5 contains the relationship among the summarized concepts in the paper and Section 6 discuss about compactness and the corresponding notions of completeness.

From now on, $\mathbb{R}, \mathbb{R}^{+}$and $\mathbb{N}$ will denote the sets of real numbers, positive real numbers and positive integers, respectively.

\section{Preliminaries}

Definition 1 (A. George, P. Veeramani [3]) A fuzzy metric space is an ordered triple $(X, M, *)$ such that $X$ is a (nonempty) set, $*$ is a continuous $t$ norm and $M$ is a fuzzy set on $X \times X \times \mathbb{R}^{+}$satisfying the following conditions, for all $x, y, z \in X, s, t>0$ :

(GV1) $M(x, y, t)>0$

(GV2) $M(x, y, t)=1$ if and only if $x=y$;

(GV3) $M(x, y, t)=M(y, x, t)$;

(GV4) $M(x, y, t) * M(y, z, s) \leq M(x, z, t+s)$;

$\left.\left.(\mathrm{GV} 5) M\left(x, y,,_{-}\right): \mathbb{R}^{+} \rightarrow\right] 0,1\right]$ is continuous.

If $(X, M, *)$ is a fuzzy metric space we say that $(M, *)$, or simply $M$, is a fuzzy metric on $X$. Also, we say that $(X, M)$ or, simply, $X$ is a fuzzy metric space. 
Let $(X, d)$ be a metric space. Denote by $a \cdot b$ the usual product for all $a, b \in$ $[0,1]$, and let $M_{d}$ be the fuzzy set defined on $X \times X \times \mathbb{R}^{+}$by

$$
M_{d}(x, y, t)=\frac{t}{t+d(x, y)}
$$

Then $\left(M_{d}, \cdot\right)$ is a fuzzy metric on $X$ called standard fuzzy metric induced by $d[3]$.

George and Veeramani proved in [3] that every fuzzy metric $M$ on $X$ generates a topology $\tau_{M}$ on $X$ which has as a base the family of open sets of the form $\left\{B_{M}(x, \varepsilon, t): x \in X, 0<\varepsilon<1, t>0\right\}$, where $B_{M}(x, \varepsilon, t)=\{y \in X:$ $M(x, y, t)>1-\varepsilon\}$ for all $x \in X, \varepsilon \in] 0,1[$ and $t>0$. In the case of the standard fuzzy metric $M_{d}$ it is well-known that the topology $\tau(d)$ on $X$ deduced from $d$ satisfies $\tau(d)=\tau_{M_{d}}$. From now on we will suppose $X$ endowed with the topology $\tau_{M}$.

Proposition 2 (A. George, P. Veeramani [3]) A sequence $\left\{x_{n}\right\}$ in a fuzzy metric space $(X, M, *)$ converges to $x_{0}$ if and only if $\lim _{n} M\left(x_{0}, x_{n}, t\right)=1$, for all $t>0$.

Definition 3 (V. Gregori, S. Romaguera [9]) A fuzzy metric $M$ on $X$ is said to be stationary if $M$ does not depend on $t$, i.e. if for each $x, y \in X$, the function $M_{x, y}(t)=M(x, y, t)$ is constant. In this case we write $M(x, y)$ instead of $M(x, y, t)$,

Definition 4 (V. Gregori, J-J. Miñana [13]) Suppose it is given a sequential stronger (weaker, respectively) concept than Cauchy sequence, say s-Cauchy sequence ( $w$-Cauchy, respectively). A concept of convergence, say s-convergence (w-convergence, respectively), is said to be compatible with s-Cauchy (w-Cauchy, respectively), and vice-versa, if the diagram of implications below on the left (on the right, respectively) is fulfilled.

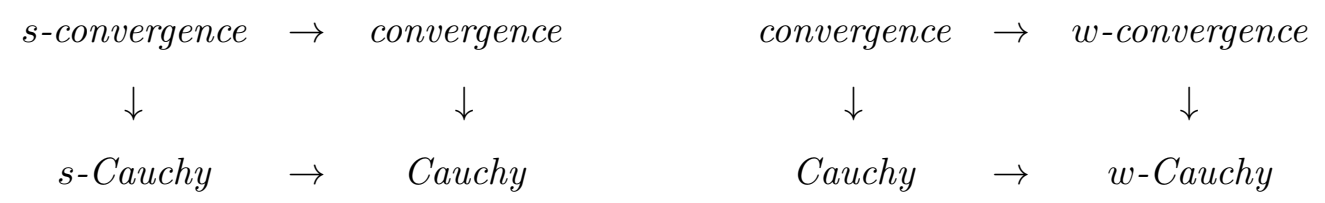

and there is not any other implication, in general, among these concepts. In such a case we also say that $w$-convergence and w-Cauchy (respectively, sconvergence and s-Cauchy) is a compatible pair. 


\section{Weak concepts of Cauchyness and convergence}

\subsection{Cauchy sequence}

The concept of Cauchy sequence which we deal with is the following.

Definition 5 (A. George, P. Veeramani [5]) A sequence $\left\{x_{n}\right\}_{n \in \mathbb{N}}$ in a fuzzy metric space $(X, M, *)$ is called Cauchy if for each $\varepsilon \in] 0,1[$ and each $t>0$ there exists $n_{0} \in \mathbb{N}$ such that $M\left(x_{n}, x_{m}, t\right)>1-\varepsilon$ for all $n, m \geq n_{0}$ or equivalently $\lim _{m, n} M\left(x_{n}, x_{m}, t\right)=1$ for all $t>0$.

$(X, M, *)$, or simply $M$, is called complete if every Cauchy sequence in $X$ is convergent with respect to $\tau_{M}$.

This concept comes, really, from the theory of $P M$-spaces and it was introduced by H. Sherwood in his theory of completion of Menger spaces [26,27]. This concept leads to extend many results of classical metric spaces to the fuzzy setting. For instance, convergent sequences are Cauchy. A closed subset of a complete fuzzy metric space is complete and complete subspaces of complete fuzzy metric spaces are closed. On the other hand, a sequence $\left\{x_{n}\right\}$ is Cauchy in a metric space $(X, d)$ if and only if $\left\{x_{n}\right\}$ is Cauchy in $\left(X, M_{d}, \cdot\right)$. Other interesting properties can be found in [7].

A drawback of this concept is that we can find fuzzy metric spaces which are not completable (for details see $[8,9]$ ). Nevertheless, every standard fuzzy metric space $\left(X, M_{d}, \cdot\right)$ admits completion [8] and $M_{d}$ is complete if and only if $d$ is complete.

\subsection{G-Cauchyness and G-convergence}

In order to establish a Banach Contraction Principle in the context of $K M$ fuzzy metric spaces, M. Grabiec gave the following weaker concept than Cauchy sequence that we denote $G$-Cauchy.

Definition 6 (M. Grabiec [6]) A sequence $\left\{x_{n}\right\}$ in a fuzzy metric space $(X, M, *)$ is called $G$-Cauchy if $\lim _{n} M\left(x_{n}, x_{n+p}, t\right)=1$ for each $t>0$ and each $p \in \mathbb{N}$.

The concept of $G$-Cauchy sequence leads to a so strong concept of $G$-completeness (Definition 35) that the usual standard fuzzy metric space $\left(\mathbb{R}, M_{d}, \cdot\right)$ is not $G$ complete (see [3], Note 3.13). A discussion on this concept can be found in [30]. On the other hand Example 9 shows, as it was observed in [28], that a compact fuzzy metric space is not necessarily $G$-complete. 
The concept of $G$-completeness has been extensively used in order to obtain fixed point theorems. For instance, in addition to the celebrated theorem due to Grabiec [6], see $[1,2,22,23,29]$. An advantage of the $G$-complete fuzzy metric spaces is that the fixed point theorems stated on them require less additional conditions, in general, than those stated on complete fuzzy metric spaces.

As it was indicated by D. Mihet [20], this definition is equivalent to $\lim _{n} M\left(x_{n}, x_{n+1}, t\right)=$ 1 for all $t>0$. If we try to define a strict weaker concept than convergence deduced in a natural way from this characterization of $G$-Cauchyness then it leads to write $\lim _{n} M\left(x_{0}, x_{n+1}, t\right)=1$ for all $t>0$. Unfortunately, this expression corresponds to the usual one of convergence. To overcome this drawback we introduce the following concept which, in some sense, involves convergence.

Definition 7 We will say that a sequence $\left\{x_{n}\right\}$ in a fuzzy metric space $(X, M, *)$ is $G$-convergent to $x_{0}$ if $\left\{x_{n}\right\}$ has a subsequence converging to $x_{0}$ (i.e., $x_{0}$ is a cluster point of $\left.\left\{x_{n}\right\}\right)$ and $\lim _{n} M\left(x_{n}, x_{n+1}, t\right)=1$ for all $t>0$.

Obviously, the next diagram of implications if fulfilled.

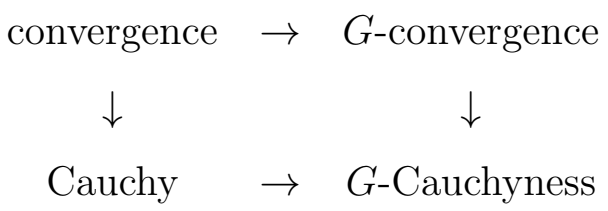

Next we give appropriate examples which prove that there are not any other implications in the last diagram and then we conclude that $G$-Cauchyness and $G$-convergence constitute a compatible pair. In these examples $M_{d}$ is the standard fuzzy metric deduced from the usual metric of $\mathbb{R}$, restricted to the corresponding sets of $\mathbb{R}$.

Example 8 (A G-Cauchy non-G-convergent non-Cauchy sequence)

Consider the sequence $\left\{x_{n}\right\}$ given by $x_{n}=\sum_{i=1}^{n} \frac{1}{i}$ defined in the fuzzy metric space $\left(\mathbb{R}, M_{d}, \cdot\right)$. It is well-known that $\left\{x_{n}\right\}$ is a $G$-Cauchy non-Cauchy sequence [5]. Clearly, $\left\{x_{n}\right\}$ is not G-convergent since it has not any cluster point.

Example 9 (A G-convergent non-Cauchy sequence)

Consider the sequence $\left\{x_{n}\right\}$ where $x_{n}=\sin \sqrt{n}$ defined in the fuzzy metric space $\left([-1,1], M_{d}, \cdot\right)$. It is easy to verifty that $\left\{x_{n}\right\}$ is $G$-Cauchy.

We will see that each point of $[-1,1]$ is a cluster point of $\left\{x_{n}\right\}$ and hence $\left\{x_{n}\right\}$ is $G$-convergent. Indeed, let $\left.y_{0} \in\right]-1,1\left[\right.$ and take $\left.t_{0} \in\right] 0,2 \pi[$ such that $\sin t_{0}=y_{0}$. Take $\varepsilon>0$ with $] y_{0}-\varepsilon, y_{0}+\varepsilon[\subset]-1,1[$ and let $N \in \mathbb{N}$. By continuity of $\sin t$ there exists $\delta>0$ such that $\left|t-t_{0}\right|<\delta$ implies $\left|\sin t-y_{0}\right|<\varepsilon$ and by 
the periodicity of $\sin t$ we have that $\left|t-t_{0}+2 k \pi\right|<\delta$ implies $\left|\sin t-y_{0}\right|<\varepsilon$ for all $k \in \mathbb{Z}^{+}$.

The sequence $\{\sqrt{n}-\sqrt{n-1}\}_{n=1}^{\infty}$ converges to 0 and then there exists $n_{0} \in \mathbb{N}$ with $n_{0}>N$ such that

$$
|\sqrt{n}-\sqrt{n-1}|<\delta \text { for all } n \geq n_{0}
$$

Let $k_{0}=\min \left\{k \in \mathbb{Z}^{+}: \sqrt{n_{0}}<t_{0}+2 k \pi\right\}$. The sequence $\left\{n_{0}+i\right\}_{i=0}^{\infty}$ tends to $+\infty$ and two consecutive terms of this sequence satisfy (1). Therefore some $i_{0} \in \mathbb{N}$ satisfies that $\left.\sqrt{n_{0}+i_{0}} \in\right] t_{0}+2 k \pi-\delta, t_{0}+2 k \pi+\delta\left[\right.$ and then $\sin \sqrt{n_{0}+i_{0}} \in$ ]$y_{0}-\varepsilon, y_{0}+\varepsilon\left[\right.$ with $n_{0}+i_{0}>N$. Therefore the sequence $\left\{x_{n}\right\}$ is frequently in ]$y_{0}-\varepsilon, y_{0}+\varepsilon\left[\right.$ and hence $y_{0}$ is a cluster point of $\left\{x_{n}\right\}$.

With slight modifications it can be proved that -1 and 1 are also cluster points of $\left\{x_{n}\right\}$.

Clearly, $\left\{x_{n}\right\}$ is not Cauchy (and consequently $\left\{x_{n}\right\}$ is not convergent).

Example 10 (A Cauchy non-G-convergent sequence)

Consider the sequence $\left\{x_{n}\right\}$ in the fuzzy metric space (] $0,+\infty\left[, M_{d}, \cdot\right)$ where $x_{n}=\frac{1}{n}$. Then $\left\{x_{n}\right\}$ is Cauchy but it is not $G$-convergent since it has not any cluster point.

\section{3 p-convergence and p-Cauchyness}

In order to obtain a fixed point theorem in a fuzzy metric space, D. Mihet gave the following weaker concept than convergence.

Definition 11 (D. Mihet [20]) A sequence $\left\{x_{n}\right\}$ in a fuzzy metric space $(X, M, *)$ is called $p$-convergent to $x_{0}$ if $\lim _{n} M\left(x_{n}, x_{0}, t_{0}\right)=1$ for some $t_{0}>0$.

This concept has been used by the authors in [11] to characterize $t$-continuous mappings. In [10] the authors continue the study started by Mihet and first they gave the following concept.

Definition 12 A fuzzy metric space $(X, M, *)$ is said to be principal (or simply, $M$ is principal) if $\{B(x, r, t): r \in] 0,1[\}$ is a local base at $x \in X$, for each $x \in X$ and each $t>0$.

It is worth to notice that many fuzzy metric spaces are principal $[10,16]$. Then the authors obtained the following characterization. 
Proposition 13 A fuzzy metric space $(X, M, *)$ is principal if and only if every $p$-convergent sequence in $X$ is convergent in $\left(X, \tau_{M}\right)$.

After, the authors of [10] gave the following concept of Cauchyness deduced in a natural way from the $p$-convergence concept.

Definition $14 A$ sequence $\left\{x_{n}\right\}$ in a fuzzy metric space $(X, M, *)$ is called $p$-Cauchy if there exists $t_{0}>0$ such that for each $\left.\epsilon \in\right] 0,1$ [ there exists $n_{0} \in \mathbb{N}$ such that $M\left(x_{n}, x_{m}, t_{0}\right)>1-\epsilon$ for all $n, m \geq n_{0}$, or equivalently, $\lim _{n, m} M\left(x_{n}, x_{m}, t_{0}\right)=$ 1 for some $t_{0}>0$.

The authors proved that the next diagram of implications is fulfilled.

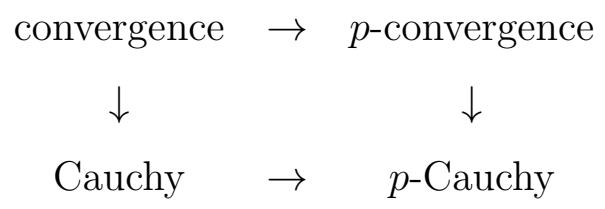

For shorten the paper, it is left to the reader to verify that there is not any other implication in such diagram and so $p$-convergence and $p$-Cauchyness is a compatible pair.

\section{Strong concepts of convergence and Cauchyness}

\subsection{Standard Cauchyness and standard convergence}

In order to establish relationships between the theory of complete fuzzy metric spaces and domain theory, the authors in [25] introduced the following stronger concept than Cauchy sequence.

Definition $15 A$ sequence $\left\{x_{n}\right\}$ in a fuzzy metric space $(X, M, *)$ is called standard Cauchy if for each $\varepsilon \in] 0,1\left[\right.$ there exists $n_{0} \in \mathbb{N}$, depending on $\varepsilon$, such that

$$
M\left(x_{n}, x_{m}, t\right)>\frac{t}{t+\varepsilon},
$$

for all $n, m \geq n_{0}$ and $t>0$.

In a standard fuzzy metric space it is fulfilled that every Cauchy sequence is standard Cauchy. Notice that the bound on the right of the above inequality depends on $t$. Consequently, a handicap of this concept is that in stationary fuzzy metric spaces one cannot find non-constant standard Cauchy sequences.

Later, the authors in [24] gave the following notion of convergence that here 
we will denote $s t d^{*}$-convergence deduced in a natural way from the standard Cauchy concept.

Definition 16 A sequence $\left\{x_{n}\right\}$ in a fuzzy metric space $(X, M, *)$ is called std ${ }^{*}$-convergent to $x_{0} \in X$ if there exists $n_{0} \in \mathbb{N}$ such that $M\left(x_{n}, x_{0}, t\right)>\frac{t}{t+\varepsilon}$ for all $n \geq n_{0}$ and $t>0$.

Now, the authors in [15] proved the existence of $s t d^{*}$-convergent sequences which are not standard Cauchy and, in consequence, st $d^{*}$-convergence is not compatible with standard Cauchy. To overcome this inconvenience, in the same paper, the authors gave the following definition.

Definition 17 A sequence $\left\{x_{n}\right\}$ is called standard convergent if it is convergent and standard Cauchy.

It was proved that this concept is compatible with standard Cauchy. One can notice that in a standard fuzzy metric space every convergent sequence is standard convergent.

\section{2 s-convergence and s-Cauchyness}

The following stronger concept than convergence, called $s$-convergence, tries to extend the classical metric formulation of convergence using a simple limit. This concept leads to define a class of fuzzy metric spaces called $s$-fuzzy metric. In an $s$-fuzzy metric space each point has a local base which is not defined by balls, but by neighbourhoods which, as in the classical case, only depend on (the radius) $r \in] 0,1\left[\right.$ (Proposition 19). Now, if $N(x, y)=\bigwedge_{t>0} M(x, y, t)>0$ for all $x, y \in X$ then $(N, *)$ is a stationary fuzzy metric on $X$ [17] and the authors ([16], Theorem 4.2) proved that $(X, M, *)$ is an $s$-fuzzy metric space if and only if $\tau_{N}=\tau_{M}$.

Definition 18 A sequence $\left\{x_{n}\right\}$ in a fuzzy metric space $(X, M, *)$ is s-convergent to $x_{0} \in X$ if $\lim _{n} M\left(x_{n}, x_{0}, \frac{1}{n}\right)=1$.

A fuzzy metric space in which every convergent sequence is $s$-convergent is called $s$-fuzzy metric space. We also say that $M$ is an $s$-fuzzy metric. $s$-fuzzy metric spaces are characterized in [16] as follows.

Proposition $19 M$ is an s-fuzzy metric if and only if $\bigcap_{t>0} B(x, r, t)$ is a neighbourhood of $x$, for all $x \in X$ and for all $r \in] 0,1[$, or equivalently, $\left\{\bigcap_{t>0} B(x, r, t): r \in\right] 0,1[\}$ is a local base at $x$, for each $x \in X$.

The corresponding concept of Cauchyness deduced in a natural way from the $s$-convergence is the following. 
Definition 20 A sequence $\left\{x_{n}\right\}$ in a fuzzy metric space $(X, M, *)$ is $s^{*}$-Cauchy if $\lim _{n, m} M\left(x_{n}, x_{m}, \frac{1}{n}\right)=1$.

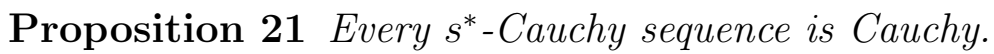

PROOF. Suppose that $\left\{x_{n}\right\}$ is $s^{*}$-Cauchy. Let $t>0$ and take $n_{0} \in \mathbb{N}$ such that $\frac{1}{n_{0}}<t$. Then we have that $M\left(x_{n}, x_{m}, t\right) \geq M\left(x_{n}, x_{m}, \frac{1}{n_{0}}\right) \geq M\left(x_{n}, x_{m}, \frac{1}{n}\right)$ for all $n \geq n_{0}$ and all $m \in \mathbb{N}$. Then $\lim _{n} M\left(x_{n}, x_{m}, t\right)=1$.

Unfortunately, an $s$-convergent sequence is not necessarily $s^{*}$-Cauchy, as shows the next example.

Example 22 Let $\mathbb{R}$ be endowed with its usual metric d. Consider the standard fuzzy metric space $\left(\mathbb{R}, M_{d}, \cdot\right)$. We will see that the sequence $\left\{x_{n}\right\}$ given by $x_{n}=\frac{1}{n^{2}}$ for all $n \in \mathbb{N}$, is s-convergent to 0 but it is not $s^{*}$-Cauchy.

We have that

$$
\lim _{n} M_{d}\left(x_{n}, 0, \frac{1}{n}\right)=\lim _{n} \frac{\frac{1}{n}}{\frac{1}{n}+\frac{1}{n^{2}}}=\lim _{n} \frac{1}{1+\frac{1}{n}}=1
$$

and thus $\left\{x_{n}\right\}$ is s-convergent to 0 .

Now, we will see that $\left\{x_{n}\right\}$ is not $s^{*}$-Cauchy. Suppose that $\left\{x_{n}\right\}$ is $s^{*}$-Cauchy, that is

$$
\lim _{n, m} M_{d}\left(x_{n}, x_{m}, \frac{1}{n}\right)=\lim _{n, m} \frac{\frac{1}{n}}{\frac{1}{n}+\left|\frac{1}{n^{2}}-\frac{1}{m^{2}}\right|}=1 .
$$

Now, for large values of $n$ and $m$, if we take $m \in \mathbb{N}$ with $m=\sqrt{n}$ we have that

$$
\lim _{n} \frac{\frac{1}{n}}{\frac{1}{n}+\left|\frac{1}{n^{2}}-\frac{1}{(\sqrt{n})^{2}}\right|}=\lim _{n} \frac{\frac{1}{n}}{\frac{1}{n}+\frac{1}{n}-\frac{1}{n^{2}}}=\lim _{n} \frac{1}{2-\frac{1}{n}}=\frac{1}{2}
$$

a contradiction.

Consequently, $s^{*}$-Cauchy is not compatible with $s$-convergence. To overcome this inconvenience, we introduce here the following definition.

Definition 23 A sequence in a fuzzy metric space $(X, M, *)$ is s-Cauchy if $\lim _{m, n} M\left(x_{n}, x_{m}, \frac{m+n}{m n}\right)=1$.

It is easy to verify that an $s$-Cauchy sequence is Cauchy. Next we will see that $s$-convergence and $s$-Cauchyness is a compatible pair.

Proposition 24 Every s-convergent sequence in a fuzzy metric space $(X, M, *)$ is s-Cauchy. 
PROOF. Let $\varepsilon \in] 0,1[$. Take $\mu \in] 0,1[$ such that $(1-\mu) *(1-\mu) \geq 1-\varepsilon$.

Let $\left\{x_{n}\right\}$ be an $s$-convergent sequence to $x_{0}$. Then there exists $n_{0} \in \mathbb{N}$ such that $M\left(x_{n}, x_{0}, \frac{1}{n}\right) \geq 1-\mu$ for all $n \geq n_{0}$. Now we have

$M\left(x_{n}, x_{m}, \frac{m+n}{m n}\right) \geq M\left(x_{n}, x_{0}, \frac{1}{n}\right) * M\left(x_{m}, x_{0}, \frac{1}{m}\right) \geq(1-\mu) *(1-\mu) \geq 1-\varepsilon$ for all $m, n \geq n_{0}$ and hence $\left\{x_{n}\right\}$ is an $s$-Cauchy sequence.

So, the following diagram of implications is fulfilled.

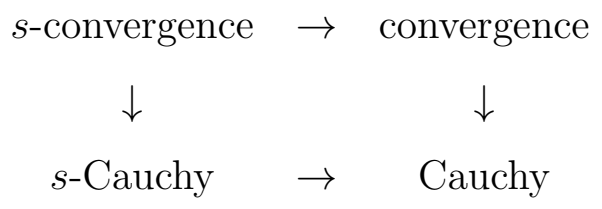

To prove that $s$-convergence and $s$-Cauchyness is a compatible pair we will see, with appropriate examples, that no other implication is fulfilled in the previous diagram.

Example 25 (An s-Cauchy non-convergent sequence)

Let $X=] 1,+\infty[$ and consider the fuzzy metric space $(X, M, \cdot)$ where $M$ is the stationary fuzzy metric [3] defined by $M(x, y)=\frac{\min \{x, y\}}{\max \{x, y\}}$.

It is easy to verify that the sequence $x_{n}=1+\frac{1}{n}$ is an s-Cauchy sequence in $X$ which is not convergent.

Example 26 (A convergent non-s-Cauchy sequence)

Let $\left(X, M_{d}, \cdot\right)$ be the standard fuzzy metric space induced by $(X, d)$ where $X=$ $\mathbb{R}$ and $d$ is the usual metric on $\mathbb{R}$.

Consider the sequence $\left\{x_{n}\right\}$ defined by $x_{n}=\frac{1}{n}$ for all $n \in \mathbb{N}$. Clearly $\left\{x_{n}\right\}$ is convergent and so it is Cauchy.

Suppose now that $\left\{x_{n}\right\}$ is an s-Cauchy sequence. Then we have that for all $\varepsilon \in] 0,1\left[\right.$ there exists $n_{\varepsilon}$ such that $M\left(x_{n}, x_{m}, \frac{m+n}{m n}\right) \geq 1-\varepsilon$ for all $n, m \geq n_{\varepsilon}$ or equivalently $\frac{\frac{m+n}{m n}}{\frac{m+n}{m n}+\left|\frac{1}{n}-\frac{1}{m}\right|}=\frac{1}{1+\left|\frac{m-n}{m+n}\right|} \geq 1-\varepsilon$ for all $m, n \geq n_{\varepsilon}$. In consequence $\left|\frac{m-n}{m+n}\right| \leq \frac{\varepsilon}{1-\varepsilon}$ for all $m, n \geq n_{\varepsilon}$, which is a contradiction. 


\subsection{Strong convergence and strong Cauchyness}

In the fuzzy context, there is another way of regarding the classical formulation of the concept of convergence using the role $\varepsilon$ and $n_{0}$ and strengthening the imposition on $t$. In fact, in [14] the authors have given the following stronger condition than $s$-convergence called strong convergence.

Definition 27 A sequence $\left\{x_{n}\right\}$ in a fuzzy metric space $(X, M, *)$ is called strong convergent to $x_{0} \in X$ if given $\left.\varepsilon \in\right] 0,1\left[\right.$ there exists $n_{\varepsilon}$, depending on $\varepsilon$, such that $M\left(x_{n}, x_{0}, t\right)>1-\varepsilon$, for all $n \geq n_{\varepsilon}$ and for all $t>0$.

The first goal of this concept is that $s$-fuzzy metric spaces are characterized because every convergent sequence is strong convergent. In the mentioned paper the authors have introduced the following concept deduced in a natural way from the concept of strong convergence.

Definition $28 A$ sequence $\left\{x_{n}\right\}$ in a fuzzy metric space $(X, M, *)$ is strong Cauchy if given $\varepsilon \in] 0,1\left[\right.$ there exists $n_{\varepsilon}$, depending on $\varepsilon$, such that $M\left(x_{n}, x_{m}, t\right)>$ $1-\epsilon$, for all $n, m \geq n_{\varepsilon}$ and for all $t>0$.

The second goal of [14] is that the authors have proved that strong convergence and strong Cauchy is a compatible pair.

\section{Relating the concepts}

\subsection{Relating weak concepts}

The only relations between the concepts appeared in this section are illustrated in the next diagram of implications.

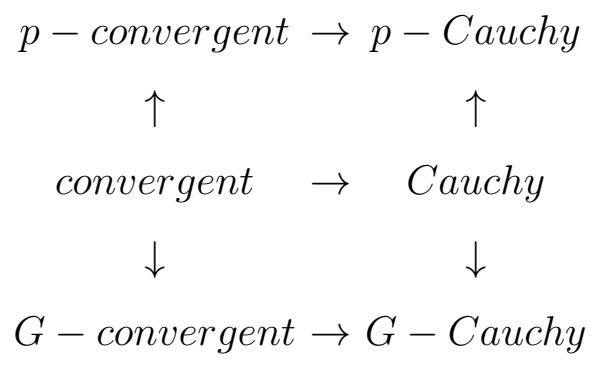

The following examples prove that there is not any other relation between these concepts. 
Example 29 (A p-Cauchy sequence which is not G-Cauchy)

Let $\left\{x_{n}\right\}$ be a strictly increasing sequence of positive real numbers converging to 1 , with respect to the usual topology of $\mathbb{R}$. Put $X=\left\{x_{n}\right\} \cup\{1\}$. Define the fuzzy set $M$ on $X^{2} \times \mathbb{R}^{+}$given by $M(x, x, t)=1$ for each $x \in X$ and $t>0$ and $M\left(x_{n}, x_{m}, t\right)=\min \left\{x_{n}, x_{m}, t\right\}$ for all $m, n \in \mathbb{N}, t>0$. Then $(M, \wedge)$ is a fuzzy metric on $X$ [20].

Consider the sequence $\left\{a_{n}\right\}$ where $a_{n}=1$ if $n$ is even and $a_{n}=x_{n}$ if $n$ is odd. It is left to the reader to verify that $\left\{a_{n}\right\}$ is p-Cauchy but it is not $G$-Cauchy.

Example 30 (A G-Cauchy sequence which is not p-Cauchy)

Consider the standard fuzzy metric space $\left(\mathbb{R}, M_{d}, \cdot\right)$ where $d$ is the usual metric on $\mathbb{R}$. Let $\left\{x_{n}\right\}$ be the sequence given by $x_{n}=\sum_{i=1}^{n} \frac{1}{i}$. It is well-known that $\left\{x_{n}\right\}$ is $G$-Cauchy. It is left to the reader to show that $\left\{x_{n}\right\}$ is not p-Cauchy.

Example 31 (A p-convergent (non-G-convergent) non-G-Cauchy sequence)

Let $X=\mathbb{R}^{+}$and let $\left.\left.\varphi: \mathbb{R}^{+} \rightarrow\right] 0,1\right]$ be a function given by $\varphi(t)=t$ if $t \leq 1$ and $\varphi(t)=1$ elsewhere. Define the function $M$ on $X^{2} \times \mathbb{R}^{+}$by

$$
M(x, y, t)=\left\{\begin{array}{cc}
1 & x=y \\
\frac{\min \{x, y\}}{\max \{x, y\}} \cdot \varphi(t) & x \neq y
\end{array}\right.
$$

It is easy to verify that $(M, \cdot)$ is a fuzzy metric on $X$ and it is obvious that the only $(G-)$ Cauchy sequences in $X$ are the constant sequences, since $M(x, y, t)<$ $t$ whenever $t \in] 0,1[$ and $x \neq y$.

Now, consider the sequence $\left\{x_{n}\right\}$ in $X$ given by $x_{n}=1-\frac{1}{n}, n \in \mathbb{N}$. We have that $M\left(x_{n}, 1,1\right)=1-\frac{1}{n}$ for all $n \in \mathbb{N}$, so $\lim _{n} M\left(x_{n}, 1,1\right)=1$ and $\left\{x_{n}\right\}$ is p-convergent to 1 , but $\left\{x_{n}\right\}$ is not G-Cauchy.

Example 32 (A G-convergent (non-p-convergent) non-p-Cauchy sequence) Consider the standard fuzzy metric space $\left([-1,1], M_{d}, \cdot\right)$ where $d$ is the usual metric on $\mathbb{R}$ restricted to $[-1,1]$. Consider the sequence $\left\{x_{n}\right\}=\sin \sqrt{n}$. In Example 9 we have seen that $\left\{x_{n}\right\}$ is $G$-convergent. It is left to the reader to verify that $\left\{x_{n}\right\}$ is not $p$-Cauchy. 


\subsection{Relating strong concepts}

The following diagram of implications shows all the relations (except convergence implies Cauchy), among all the concepts summarized in this section.

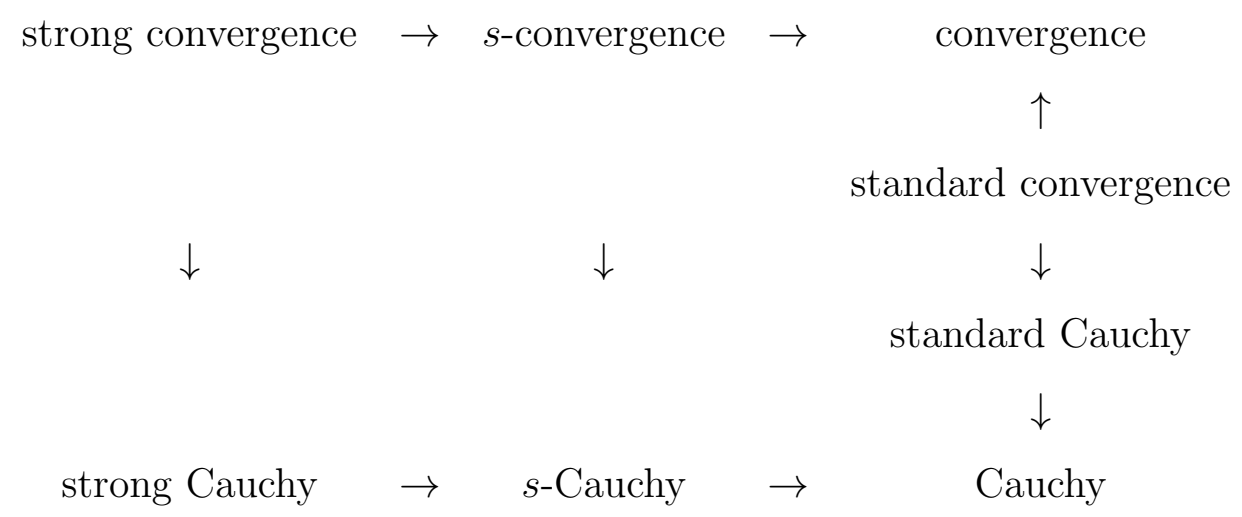

The following examples (jointly with the results of Section 4) show that there is not any other implication among these concepts.

Example 33 (A strong convergent non-standard Cauchy (non-standard convergent) sequence)

Consider the fuzzy metric space $(X, M, *)$ where $X=[1,+\infty[, M(x, y, t)=$ $\frac{\min \{x, y\}+\sqrt{t}}{\max \{x, y\}+\sqrt{t}}$ and $*$ is the usual product on $[0,1][18]$.

Consider the sequence $\left\{x_{n}\right\}$ where $x_{n}=1+\frac{1}{n}, n \in \mathbb{N}$. It is left to the reader to verify that $\left\{x_{n}\right\}$ is strong convergent (to 1) and it is not standard Cauchy.

Example 34 (A standard convergent (non-s-convergent) non-s-Cauchy sequence)

Consider the standard fuzzy metric space $\left(\mathbb{R}, M_{d}, \cdot\right)$ where $d$ is the usual metric on $\mathbb{R}$. The sequence $\left\{x_{n}\right\}$ where $x_{n}=\frac{1}{n}, n \in \mathbb{N}$ is standard convergent to 0 . It is left to the reader to show that it is not s-Cauchy.

\section{Compactness and completeness}

In this section we study the relationship between compactness and all the concepts of completeness based on the above Cauchy concepts.

Definition 35 We will say that a fuzzy metric space $(X, M, *)$, or simply $M$, is p-complete if every $p$-Cauchy sequence in $X$ converges in $\left(X, \tau_{M}\right)$. This 
definition is extended to the other concepts of Cauchyness appeared before.

We have seen that compactness does not imply $G$-completenes. Now we will prove that every compact space is $p$-complete. We start with the following lemma.

Lemma 36 (V. Gregori et al. [10]) Let $\left\{x_{n}\right\}$ be a p-convergent sequence to $x_{0}$. Then:

(i) Each subsequence $\left\{x_{n_{k}}\right\}$ of $\left\{x_{n}\right\}$ is p-convergent to $x_{0}$.

(ii) If $\left\{x_{n}\right\}$ is convergent, then it converges to $x_{0}$.

Theorem 37 Compact fuzzy metric spaces are principal.

PROOF. let $(X, M, *)$ be a compact fuzzy metric space. Suppose that $X$ is not principal. Then there exist $x_{0} \in X$ and $t_{0}>0$ such that the family of open balls $\left\{B\left(x_{0}, \frac{1}{n}, t_{0}\right): n \in \mathbb{N}\right\}$ is not a local base at $x_{0}$, that is, there exist $\left.r \in\right] 0,1[$ and $t>0$ such that for all $n \in \mathbb{N}$ it is satisfied $B\left(x_{0}, \frac{1}{n}, t_{0}\right) \nsubseteq B\left(x_{0}, r, t\right)$.

By induction we construct a sequence $\left\{x_{n}\right\}$ where $x_{n} \in B\left(x_{0}, \frac{1}{n}, t_{0}\right) \backslash B\left(x_{0}, r, t\right)$ for all $n>1$. By construction $\left\{x_{n}\right\}$ is not convergent and also $\lim _{n} M\left(x_{0}, x_{n}, t_{0}\right)=$ 1 and hence $\left\{x_{n}\right\}$ is $p$-convergent to $x_{0}$.

Since $X$ is compact we can find a subsequence $\left\{x_{n_{k}}\right\}$ of $\left\{x_{n}\right\}$ which converges in $X$. By (i) of Lemma 36, $\left\{x_{n_{k}}\right\}$ is $p$-convergent to $x_{0}$ and by (ii) $\left\{x_{n_{k}}\right\}$ converges to $x_{0}$, a contradiction since $x_{n} \notin B\left(x_{0}, r, t\right)$ for all $n \in \mathbb{N}$. So, $X$ is principal.

Corollary 38 Compact fuzzy metric spaces are p-complete.

PROOF. let $(X, M, *)$ be a compact fuzzy metric space and let $\left\{x_{n}\right\}$ be a $p$-Cauchy sequence in $X$. Since $X$ is compact we can find a subsequence $\left\{x_{n_{k}}\right\}$ of $\left\{x_{n}\right\}$ which converges to $x_{0} \in X$. Suppose that $\lim _{n} M\left(x_{n}, x_{m}, t_{0}\right)=1$ for some $t_{0}>0$ since $\left\{x_{n}\right\}$ is $p$-Cauchy. Then we have

$$
M\left(x_{n}, x_{0}, 2 t_{0}\right) \geq M\left(x_{n}, x_{n_{k}}, t_{0}\right) * M\left(x_{n_{k}}, x_{0}, t_{0}\right)
$$

and taking limit as $n$ and $k$ tend to $+\infty$ we obtain $\lim _{n} M\left(x_{n}, x_{0}, 2 t_{0}\right)=1$ and so $\left\{x_{n}\right\}$ is $p$-convergent to $x_{0}$. Now, by Theorem $37\left\{x_{n}\right\}$ is convergent and hence $X$ is $p$-complete.

Remark 39 The converse of Corollary 38 is not true. Indeed, it is easy to verify that $\left(\mathbb{R}, M_{d}, \cdot\right)$, where $d$ is the usual metric on $\mathbb{R}$, is $p$-complete and it is not compact. 
As a consequence of the last paragraphs and due to the relationship among the concepts of Cauchyness given in Sections 3-4 we have the following diagram of implications.

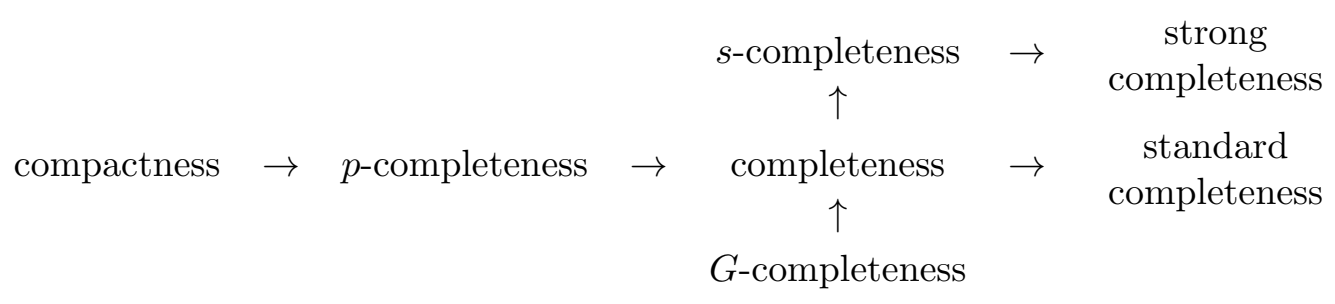

\section{References}

[1] C. Alaca, D. Turkoglu, C. Yildiz, Fixed points in intuitionistic fuzzy metric spaces, Chaos, Solitons and Fractals 29 (2006) 1073-1078.

[2] J.X. Fang, On fixed point theorems in fuzzy metric spaces, Fuzzy Sets and Systems 46-1 (1992) 107-113.

[3] A. George, P. Veeramani, On some results in fuzzy metric spaces, Fuzzy Sets and Systems 64 (1994), 395-399.

[4] A. George, P. Veeramani, Some theorems in fuzzy metric spaces, J. Fuzzy Math. 3 (1995) 933-940.

[5] A. George, P. Veeramani, On some results of analysis for fuzzy metric spaces, Fuzzy Sets and Systems 90 (1997) 365-368.

[6] M. Grabiec, Fixed points in fuzzy metric spaces, Fuzzy Sets and Systems 27 (1989) 385-389.

[7] V. Gregori, S. Romaguera, Some properties of fuzzy metric spaces, Fuzzy Sets and Systems 115 (2000) 485-489.

[8] V. Gregori, S. Romaguera, On completion of fuzzy metric spaces, Fuzzy Sets and Systems 130 (2002) 399-404.

[9] V. Gregori, S. Romaguera, Characterizing completable fuzzy metric spaces, Fuzzy Sets and Systems 144 (2004) 411-420.

[10] V. Gregori, A. Lï $₫ \frac{1}{2}$ pez-Crevillï $i \frac{1}{2} \mathrm{n}$, S. Morillas, A. Sapena, On convergence in fuzzy metric spaces, Topology and its Applications 156 (2009) 3002ï $\frac{1}{2} 3006$.

[11] V. Gregori, J.J. Miñana, Some concepts realted to continuity in fuzzy metric spaces, Proceedings of the Conference in Applied Topology WiAT'13, (2013), 85-91.

[12] V.Gregori, J-J. Miñana, A. Sapena On Banach contraction principles in fuzzy metric spaces, submitted. 
[13] V. Gregori, J-J. Miñana, std-Convergence in fuzzy metric spaces, Fuzzy Sets and Systems (2014), in press. DOI: 10.1016/j.fss.2014.05.007.

[14] V. Gregori, J-J. Miñana, Strong convergence in fuzzy metric spaces, Filomat, accepted.

[15] V. Gregori, J-J. Miñana, S. Morillas, Some questions in fuzzy metric spaces, Fuzzy Sets and Systems 204 (1) (2012) 71-85.

[16] V. Gregori, J-J. Miñana, S. Morillas, A note on convergence in fuzzy metric spaces, Iranian Journal of Fuzzy Systems, 11(4) (2014) 75-85.

[17] V. Gregori, S. Morillas, A. Sapena, On a class of completable fuzzy metric spaces, Fuzzy Sets and Systems 161 (2010) 2193-2205.

[18] V. Gregori, S. Morillas, A. Sapena, Examples of fuzzy metric spaces and applications, Fuzzy Sets and Systems 170 95-111 (2011).

[19] I. Kramosil, J. Michalek, Fuzzy metric and statistical metric spaces, Kybernetika 11 (1975) 326-334.

[20] D. Mihet, On fuzzy contractive mappings in fuzzy metric spaces, Fuzzy Sets and Systems 158 (2007) 915-921.

[21] D. Mihet, Fuzzy $\varphi$-contractive mappings in non-Archimedean fuzzy metric spaces, Fuzzy Sets and Systems 159 (2008) 739-744.

[22] D. Mihet, A Banach contraction theorem in fuzzy metric spaces, Fuzzy Sets and Systems 144 (2004) 431-439.

[23] S.N. Mishra, N. Sharma, S.L. Singh, Common fixed points of maps on fuzzy metric spaces Internat. J. Math. \& Math. Sci. 17-2 (1994) 253-258.

[24] S. Morillas, A. Sapena, On Cauchy sequences in fuzzy metric spaces, Proceedings of the Conference in Applied Topology (WiATi $\left.; \frac{1}{2} 13\right)$, (2013) 101108.

[25] Luis A. Ricarte, Salvador Romaguera, A domain-theoretic approach to fuzzy metric spaces, Topology and its Applications, 163 (2014) 149-159.

[26] H. Sherwood, On the completion of probabilistic metric spaces, Z.Wahrscheinlichkeitstheorie verw. Geb. 6 (1966) 62-64.

[27] H. Sherwood, Complete Probabilistic Metric Spaces, Z. Wahrscheinlichkeitstheorie verw. Geb. 20 (1971) 117-128.

[28] P. Tirado, On compactness and G-completeness in fuzzy metric spaces, Iranian Journal of Fuzzy Systems 9 (4) (2012) 151-158.

[29] P. Tirado, Contraction mappings in fuzzy quasi-metric spaces and [0,1]-fuzzy posets, Fixed Point Theory 13-1 (2012) 273-283.

[30] R. Vasuki, P. Veeramani, Fixed point theorems and Cauchy sequences in fuzzy metric spaces, Fuzzy Sets and Systems 135-3 (2003) 415-417. 
[31] P. Veeramani, Best approximation in fuzzy metric spaces, J. Fuzzy Math. 9 (2001) 75-80. 